\section{KOMPASS}

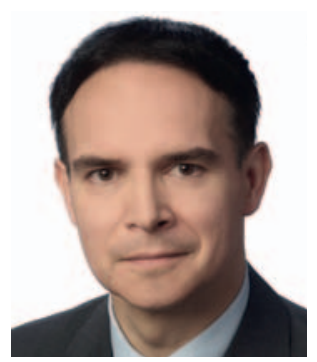

Lars-Olof Hattenbach

Klinik für Augenheilkunde,

Klinikum Ludwigshafen

\title{
An der Schwelle zur Zukunft: Neue Technologien in der Augenheilkunde
}

Neue Technologien bestimmen seit jeher den Wandel der modernen Augenheilkunde. Techniken, die uns seit Jahren vertraut und aus dem klinischen Alltag kaum noch wegzudenken sind, können schon morgen ihren Stellenwert verlieren oder werden langsam, aber stetig durch völlig neue Ansätze ersetzt. Dabei geschieht dies nicht immer ohne kontrovers geführte Diskussionen über die Vor- und Nachteile der neuen Technologien im Vergleich zu den etablierten Verfahren, zumal in Zeiten knapper Budgets immer auch die Frage nach dem tatsächlichen Nutzen einer Methode gestellt wird. Prominente Beispiele hierfür sind die Entwicklung der Femtolaser-assistierten Kataraktchirurgie als Alternative zur manuellen Phakoemulsifikation, die dreidimensionale «Heads-Up»-Operationsmikroskopie oder die intraoperative optische Echtzeit-Kohärenztomografie (iOCT). Doch während sich dem Anwender zumindest die Vorteile chirurgischer Präzision und Sicherheit hinsichtlich der neuen Technologien sofort erschließen, ist der diagnostische Nutzen aus der Weiterentwicklung bereits verfügbarer bildgebender Verfah- ren mitunter erst auf den zweiten Blick erkennbar. Dass mit diesen Verfahren bereits jetzt spektakuläre Einblicke in bisher verborgene Strukturen des Auges möglich sind, zeigt die Übersichtsarbeit von Huang et al. [1] in der vorliegenden Ausgabe des Karger Kompass Ophthalmologie. Eine exaktere Darstellung kapillärer Veränderungen bei vaskulären Erkrankungen der Netzhaut oder eine genauere Klassifizierung choroidaler Neovaskularisationen bei exsudativer AMD kann zu einem besseren Verständnis und einer gezielteren Therapie dieser Krankheitsbilder beitragen.

Der Traum vom «künstlichen Sehen» und die Überwindung der Retinitis pigmentosa als einer unheilbaren, zur Erblindung führenden Erkrankung stellt sicher einen der faszinierendsten zukunftsorientierten Ansätze der Augenheilkunde überhaupt dar. Im Spotlight der aktuellen Ausgabe des Karger Kompass Ophthalmologie [2] stellt Eberhard Zrenner, einer der bedeutendsten Experten unserer Zeit auf dem Gebiet der Retinitis-pigmentosa-Forschung, Fortschritte in der Therapie dieser Erkrankung vor. Mittlerweile gibt es zunehmend Erfahrungen in der Entwicklung der nächsten Generation von Retina-Implantaten im Rahmen der «Electronic retinal implant surgery». In jüngerer Zeit wurden zudem Ergebnisse zur Anwendung nichtinvasiver Behandlungsmethoden wie der transkornealen elektrischen Stimulation (TES) veröffentlicht [3], mit der offenbar im ERG darstellbare Verbesserungen erzielt werden können.

Mit den Beiträgen zum Fokus «Neue Technologien in der Augenheilkunde» wagt Karger Kompass Ophthalmologie einen faszinierenden Blick in die Zukunft unseres Fachs. Darüber hinaus präsentiert die aktuelle Ausgabe natürlich auch den Wissenstransfer aus der aktuellen Forschung, mit dem wir Ihnen eine für den Klinik- und Praxisalltag hilfreiche Auswahl an Inhalten aus den Publikationen des Karger-Verlags vorstellen wollen.

$\mathrm{Ihr}$

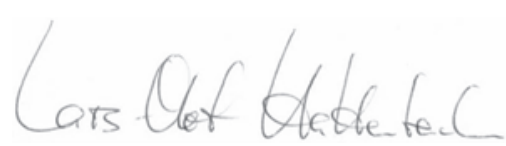

Lars-Olof Hattenbach

\section{Literatur}

1 Huang D, Jia Y, Gao SS, et al.: Optische Kohärenztomographie-Angiographie mit dem Optovue-System. Karger Kompass Ophthalmol 2017;3:00-00.
2 Zrenner E: Beiträge zu Behandlungsmöglichkeiten für Patienten mit erblichen Netzhautdystrophien. Karger Kompass Ophthalmol 2017;3:72-74.
3 Schatz A, Pach J, Gosheva M, et al.: Transcorneal electrical stimulation for patients with retinitis pigmentosa: a prospective, randomized, sham-controlled follow-up study over 1 year. Invest Ophthalmol Vis Sci. 2017;58: 257-269.

\section{KARGER}

Fax +497614520714 information@karger.com www.karger.com (c) 2017 S. Karger GmbH, Freiburg
Prof. Dr. Lars-Olof Hattenbach, FEBO

Klinik für Augenheilkunde

Klinikum Ludwigshafen

Bremserstraße 79, 67063 Ludwigshafen, Deutschland

hattenbach.LO@klilu.de 


\section{FOKUS}

\section{Neue Technologien in der Ophthalmologie}

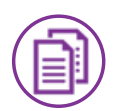

Hintergrund

Die apparativen diagnostischen Techniken in der Ophthalmologie entwickeln sich in letzten Jahren rasant. Diese Entwicklung ermöglicht uns, neue Horizonte in der Diagnostik zu erschließen und Augenerkrankungen früher zu erkennen bzw. besser zu kontrollieren. Gleichzeitig werden auch operative Techniken immer weniger invasiv. Das führt dazu, dass Behandlungen inzwischen auch bei Patienten möglich sind, bei denen größere Eingriffe Schwierigkeiten bereiten. Des Weiteren erlauben minimalinvasive Operationstechniken eine schnelle Rehabilitation und verkürzen damit die Gesamtdauer von krankheitsbedingten Einschränkungen.

\section{Herausforderungen und Handlungsbedarf}

Die Einführung der neuen diagnostischen und therapeutischen Methoden ist in der Regel nicht nur kostenintensiv, sondern verlangt oft auch zusätzliche Energie und Zeit, um diese Techniken zu erlernen. Daraus ergibt sich die berechtigte Frage, ob alle Praxen, Zentren oder Kliniken über ein volles Spektrum dieser innovativen Methoden verfügen müssen. Durch die zunehmende Subspezialisierung ist es sicherlich sinnvoll, wenn die Zentren, die neue operative Methoden in einem gewissen Gebiet anbieten, auch die entsprechende innovative Diagnostik für diese Erkrankungen durchführen. Bei einigen Methoden wird sich sicherlich in absehbarer Zeit zeigen, ob sich diese Techniken dauerhaft durchsetzen werden und ob sie somit zu einem unverzichtbaren Bestandteil im klinischen Alltag werden.

\section{Neue Technologien - ein Überblick}

Zunehmend erscheinen neue Publikationen und Studienergebnisse, die die Bedeutung der Weitwinkeldarstellung der Netzhaut in der klinischen Routine belegen. Bei vielen Netzhauterkrankungen, bei denen die Sehkraft durch ein Makulaödem beeinträchtigt wird, werden in Weitwinkelaufnahmen Veränderungen in der äußeren Netzhautperipherie gefunden. Bei Patienten mit diabetischer Retinopathie oder retinalem Venenverschluss wird seit längerem eine Korrelation zwischen dem Makulaödem und der Ausdehnung der peripheren Minderperfusion diskutiert. Jedoch steht eine eindeutige Evidenz für die Laserphotokoagulation der Netzhautperiphere zur Reduktion des Makulaödems oder zur Verringerung der Frequenz der Injektionstherapie derzeit noch aus. Um hierzu eine klare Empfehlung geben zu können, müssen die Ergebnisse weiterer Studien abgewartet werden.

\section{Optische Kohärenztomographie (OCT)-Angiographie}

Diese sehr junge Technologie eröffnet neue Horizonte in der nichtinvasiven Darstellung der Netzhautdurchblutung [1-3], wenngleich noch technische Limitationen bestehen und die Interpretation der erstellten Bilder erst standardisiert werden muss.

Die intraoperative OCT-Technik kombiniert die Eigenschaften der stark vergrößernden chirurgischen Mikroskopie mit der Auflösung der OCT und ermöglicht dadurch eine intraoperative Echtzeitbildgebung [4]. Damit liefert sie während des chirurgischen Eingriffs ergänzende und oft sehr wertvolle Informationen, die die Präzision der Operation und damit die Sicherheit für den Patienten erhöhen [4]. Jedoch ist der Stellenwert dieser Technologie im praktischen Alltag in Anbetracht der nicht unerheblichen Anschaffungskosten sowie des intraoperativen Mehraufwands - auch auf die Konzentration des Operateurs bezogen - noch unklar.

\section{Mikroinvasive Chirurgie}

Die nahtlose Vitrektomie-Technik (23/25/27 Gauge-Vitrektomie) gehört unumstritten zur Routine. Die OP-Zeit und das OP-Trauma werden verringert und damit die Belastung sowie die Beschwerden des Patienten.

Eine ähnliche Entwicklung kann derzeit auch in der Glaukomchirurgie beobachtet werden. Die klassischen filtrierenden OP-Techniken werden rasant durch eine Reihe minimalinvasiver Methoden ergänzt. Zu diesen OP-Verfahren gehören I-Stents oder Xen-Technik. Beide stellen bei Patienten mit unzureichender Druckeinstellung unter konservativer Tropftherapie eine Möglichkeit dar, die gewünschte Druckregulation ohne wesentliche intra- und postoperative Belastungen zu erreichen. Allerdings fehlt es auch hier noch an Langzeitergebnissen. Erst diese werden die Rolle dieser Methoden in der Glaukombehandlung eindeutig zeigen.

\section{Femtosekundenlaser}

Der Vorteil der Femtosekundenlaser-Technologie bei refraktiven Eingriffen ebenso wie in der Kataraktchirurgie ist die sehr präzise minimale Schnittfläche. Durch sehr detaillierte Planung können die individuellen Gegebenheiten berücksichtigt werden, um das Endergebnis zu optimieren. Die Langzeitrisiken der Technologie sind aber derzeit nur wenig erforscht.

\section{$\circledast$ Literatur}

1 Feltgen N: Optische Kohärenztomographie-Angiographie der fovealen avaskulären Zone bei retinalem Venenverschluss. Karger Kompass Ophthalmol 2016;2:78-79.

2 Lommatzsch A, Farecki ML, Book B, et al.: OCT-Angiographie bei der exsudativen altersbedingten Makuladegeneration. Ophthalmologe 2016; 113:23-29.

3 Coscas G, Lupidi M, Coscas F: Heidelberg Spectralis optical coherence tomography angiography: technical aspects. Dev Ophthalmol 2016;56:1-5.

4 Siebelmann S, Bachmann B, Matthaei M, et al.: Intraoperative Optische Kohärenztomographie. Z prakt Augenheilkd 2016;37:462-466.

Prof. Dr. Matus Rehak, Universitätsklinikum Leipzig AöR, Geschäftsführender Oberarzt der Klinik und Poliklinik für Augenheilkunde 